\title{
Some Estimates of the Coefficients in the Chebyshev Series Expansion of a Function
}

\section{By David Elliott and George Szekeres}

1. Introduction. If $f(x)$ is of bounded variation in $-1 \leqq x \leqq 1$, then we can expand $f(x)$ in a convergent series of Chebyshev polynomials $T_{n}(x)$ as

$$
f(x)=\sum_{n=0}^{\infty} a_{n} T_{n}(x),
$$

where $T_{n}(x)=\cos (n \operatorname{arc} \cos x)$, and $\sum^{\prime}$ denotes a sum whose first term is halved. In this paper we shall consider two problems. The first concerns finding an asymptotic estimate for large $n$ of the coefficients $a_{n}$ when $f(z)(z=x+i y)$ is an integral function. This problem has been previously considered by one of us [1] for more general $f(z)$. In particular, asymptotic estimates were found for $a_{n}$ in the cases when $f(z)$ has (1) simple poles, (2) a pole of order $k$, and (3) branch points. For the case when $f(z)$ is an integral function, only an upper bound for $\left|a_{n}\right|$ was given. This result is not considered to be very satisfactory. In Sections 2 and 3, we shall consider this problem again, using the method of steepest descents to give an asymptotic estimate for $a_{n}$.

In the second part of the paper (Sections 4-6), we shall consider the Chebyshev expansion of $f(x)$ given its Laplace transform $\bar{f}(p)$. In the use of Chebyshev series for the numerical solution of differential and integral equations (see, for example, [2] and [3]), it is convenient to have an a priori estimate for the coefficients $a_{n}$ in the Chebyshev expansion of the unknown function. In certain cases it is possible to evaluate the Laplace transform of the solution, and it is then desirable to estimate: $a_{n}$ directly from this transform. We shall obtain an expression for $a_{n}$ in terms of $\bar{f}(p)$ which can be used either for asymptotic evaluation or, if $\bar{f}(p)$ is sufficieritly simple, to determine $a_{n}$ explicitly. In Section 6 we shall give examples of expansions which have been obtained in this manner.

2. Integral Functions. It has been shown in [1], that the coefficient $a_{n}$ can be expressed as a contour integral,

$$
a_{n}=\frac{1}{\pi i} \int_{C} \frac{f(z) d z}{\sqrt{ }\left(z^{2}-1\right) \cdot\left(z+\sqrt{ }\left(z^{2}-1\right)\right)^{n}} \text { for } n \geqq 0,
$$

where $C$ is any contour enclosing the interval $-1 \leqq x \leqq 1$, on and within which $f(z)$ is regular. When $f(z)$ is an integral function, the contour $C$ can be displaced freely in the complex plane, provided only that it never crosses the branch points \pm 1 . This suggests the use of the method of steepest descents in order to estimate $a_{n}$, where the contour is deformed to pass through the saddle points of the integrand (see, for example, [4]). If the integrand is written as $\exp \psi(z)$, we find that a suit-

Received March 11, 1964. This research was supported in part by the U. S. Air Force Office of Scientific Research under contract number AF-AFOSR-63-300. 
able portion of the integral passing through a saddle point (where $\psi^{\prime}(\zeta)=0$ ) is asymptotically equal to

$$
\sqrt{ }(2 \pi) \alpha\left|\psi^{\prime \prime}(\zeta)\right|^{-1 / 2} \exp [\psi(\zeta)]
$$

where $\alpha$ is a complex number defined by

$$
|\alpha|=1, \quad \arg \alpha=\frac{1}{2} \pi-\frac{1}{2} \arg \psi^{\prime \prime}(\zeta) .
$$

From equation (2), we write

$$
\psi(z)=\log f(z)-\frac{1}{2} \log \left(z^{2}-1\right)-n \log \left(z+\sqrt{ }\left(z^{2}-1\right)\right),
$$

and the saddle point $\zeta$ is given by

$$
\frac{f^{\prime}(\zeta)}{f(\zeta)}=\frac{\zeta}{\zeta^{2}-1}+\frac{n}{\sqrt{ }\left(\zeta^{2}-1\right)}
$$

(There may, of course, be several values of $\zeta$ satisfying this equation.) Differentiating equation (5) a second time gives

$$
\psi^{\prime \prime}(\zeta)=\frac{f^{\prime \prime}(\zeta)}{f(\zeta)}-\left[\frac{f^{\prime}(\zeta)}{f(\zeta)}\right]^{2}+\frac{\zeta^{2}+1}{\left(\zeta^{2}-1\right)^{2}}+\frac{n \zeta}{\left(\zeta^{2}-1\right)^{3 / 2}}
$$

If it is possible to choose the contour $C$ so that the contribution of the remainder of the integral is negligible when $n$ is large, then we have

$$
a_{n} \sim \frac{1}{i} \sqrt{\left(\frac{2}{\pi}\right)} \frac{\alpha f(\zeta)}{\sqrt{ }\left(\left(\zeta^{2}-1\right)\left|\psi^{\prime \prime}(\zeta)\right|\right) \cdot\left(\zeta+\sqrt{ }\left(\zeta^{2}-1\right)\right)^{n}}
$$

for large $n$, or a sum of such expressions if the contour passes through several saddle points. This is the required asymptotic formula.

For the purposes of this formula, it is not necessary to solve equation (6) exactly. A sufficiently good approximation to $\zeta$ is obtained, for large $n$, if we omit the first term of the right-hand side of equation (6) and solve the simpler equation

$$
\sqrt{ }\left(\zeta^{2}-1\right) \frac{f^{\prime}(\zeta)}{f(\zeta)}=n
$$

In the next section, we shall illustrate the method by considering the functions $\exp (k x)$ and erf $(k x)$.

3. Two Examples. Let us considf: first the function $\exp (k x)$, where $k>0$. We assume that $n$ is large compared with $k$. From equation (9) we have $\zeta=\sqrt{ }\left(1+n^{2} / k^{2}\right)$, and from $(7), \psi^{\prime \prime}(\zeta) \sim k^{2} / n$ for $n / k$ large. With this value of $\psi^{\prime \prime}(\zeta)$, equation (4) gives immediately that $\alpha=i$. Finally,

$$
f(\zeta)=\exp \left(\sqrt{ }\left(k^{2}+n^{2}\right)\right)
$$

and

$$
\left(\zeta+\sqrt{ }\left(\zeta^{2}-1\right)\right)^{n}=k^{-n}\left(n+\sqrt{ }\left(n^{2}+k^{2}\right)\right)^{n} .
$$

We choose, as our contour $C$, a circle with centre at the origin and of radius $\zeta$. Then equation (8) gives

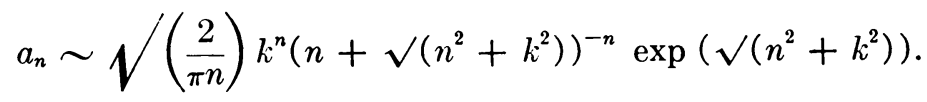


If $k^{3} / n^{2}$ is small, then on using Stirling's formula, this simplifies to

$$
a_{n} \sim \frac{k^{n}}{2^{n-1} n !} e^{k^{2} / 4 n}
$$

For our second example, we consider the function erf $(k x)=(2 / \sqrt{ }(\pi))$. $\int_{0}^{k x} e^{-t^{2}} d t$. We find that there are now two saddle points at $\zeta= \pm i \rho$, where $\rho$ is very nearly the positive solution of the equation

$$
\rho \sqrt{ }\left(\rho^{2}+1\right)=n / 2 k^{2} .
$$

This is obtained from equation (9), and using the relation that

$$
e^{-x^{2}} \int_{0}^{x} e^{t^{2}} d t \sim \frac{1}{2 x}+\frac{1}{4 x^{3}}+\cdots \quad \text { for large } x .
$$

Let us consider first the point $\zeta_{1}=+i \rho$. Writing $\rho=\sinh \beta$, equation (12) gives

$$
\sinh 2 \beta=n / k^{2} \text {, }
$$

and from equations (7), (13) and (14) we obtain

$$
\psi^{\prime \prime}\left(\zeta_{1}\right) \sim-2 k^{2}\left(1+\tanh ^{2} \beta\right) .
$$

Furthermore,

$$
\zeta_{1}+\sqrt{ }\left(\zeta_{1}^{2}-1\right)=i \exp (\beta)
$$

and

$$
\operatorname{erf}\left(k \zeta_{1}\right) \sim \frac{i \exp ((n / 2) \tanh \beta)}{(\sqrt{ } \pi) k \sinh \beta} .
$$

We choose, as our contour $C$, a circle of radius $\rho$ and centre at the origin. Since this contour is described in the positive direction, we choose $\alpha_{1}=-1$. If $a_{n}^{(j)}, j=1,2$, denotes the contribution to $a_{n}$ of the saddle point $\zeta_{j}$, then we find from equation (8) that

$$
a_{n}^{(1)} \sim \frac{2}{\pi n} \frac{\exp [(n / 2)(\tanh \beta-2 \beta)]}{\left(1+\tanh ^{2} \beta\right)^{1 / 2}} \cdot \frac{1}{i^{n-1}} .
$$

A similar result is found for ${a_{n}}^{(2)}$, but with $i^{n-1}$ written in place of $1 / i^{n-1}$. Combining the two results we find, when $n$ is odd $(=2 m+1)$,

$$
a_{2 m+1} \sim \frac{4(-1)^{m} \exp [(m+(1 / 2))(\tanh \beta-2 \beta)]}{\pi(2 m+1)\left(1+\tanh ^{2} \beta\right)^{1 / 2}},
$$

where

$$
\sinh 2 \beta=(2 m+1) / k^{2} .
$$

When $n$ is even $(=2 m), a_{2 m} \cong 0$. Equation (16) may be simplified if we assume that $2 m+1$ is large compared with $k^{2}$, to give

$$
a_{2 m+1} \sim \frac{(2 \sqrt{ } 2)(-1)^{m}}{\pi(2 m+1)}\left(\frac{k^{2} e}{2(2 m+1)}\right)^{m+1 / 2} e^{-k^{2} / 2} .
$$


TABLE 1

The coefficients $a_{2 m+1}$

\begin{tabular}{c|c|c}
\hline$m$ & Asymptotic & Actual \\
\hline 0 & +1.25290 & +1.25284 \\
7 & -0.002889 & -0.002881 \\
14 & $+0.0^{6} 5664$ & $+0.0^{6} 5654$ \\
21 & $-0.0^{117304}$ & $-0.0^{117298}$ \\
28 & $+0.0^{16} 1232$ & $+0.0^{16} 1232$ \\
\hline
\end{tabular}

The asymptotic values of $a_{2 m+1}$ as given by equation (16), and the actual values as given by Clenshaw [5], for $k=4$, are shown in Table 1 for $m=0(7) 28$. The relative error is less than $0.3 \%$ for all $m$.

4. Laplace Transform; Expansion in $T_{n}{ }^{*}(x)$. We now turn to our second problem, the determination of the coefficients in the Chebyshev series expansion of a function, given its Laplace transform $\bar{f}(p)$. As a preliminary step we consider the expansion of $f(x)$ in a series of shifted Chebyshev polynomials $T_{n}{ }^{*}(x)$,

$$
f(x)=\sum_{n=0}^{\infty} A_{n} T_{n}^{*}(x), \quad 0 \leqq x \leqq 1,
$$

where $T_{n}{ }^{*}(x)=\cos n \theta, 2 x-1=\cos \theta$.

The Laplace transform $\bar{f}(p)$ of $f(x)$ is defined by

$$
\bar{f}(p)=\int_{0}^{\infty} e^{-p x} f(x) d x .
$$

Under suitable regularity conditions the well-known inversion formula gives

$$
f(x)=\frac{1}{2 \pi i} \lim _{\beta \rightarrow \infty} \int_{c-i \beta}^{c+i \beta} e^{p x} \bar{f}(p) d p,
$$

where the real number $c$ is chosen so that all singularities of $\bar{f}(p)$ are to the left of the line of integration. The inversion formula is valid for $x>0$ and, in particular, for $x$ in the interval $(0,1]$. Now, for $0 \leqq x \leqq 1$,

$$
e^{p x}=2 \sum_{n=0}^{\infty} e^{p / 2} I_{n}(p / 2) T_{n}{ }^{*}(x),
$$

where $I_{n}$ denotes the modified Bessel function of the first kind of order $n$. Substituting equations (18) and (21) into (20), and equating coefficients, we have at once that

$$
A_{n}=\frac{1}{\pi i} \lim _{\beta \rightarrow \infty} \int_{c-i \beta}^{c+i \beta} e^{p / 2} I_{n}(p / 2) \bar{f}(p) d p \quad \text { for } n \geqq 0 .
$$

Depending on the form of $\bar{f}(p)$, the integral in equation (22) may be evaluated either exactly or asymptotically for large $n$, by suitably displacing the path of integration. The following is a useful result. 
Theorem 1. Suppose that $\bar{f}(p)$ is uniform in the left half-plane $L_{c}=$ $\{p \mid \operatorname{Re} p \leqq c\}$ and that

$$
\lim p^{1 / 2} \vec{f}(p)=0
$$

when $p \rightarrow \infty$ in $L_{c}$.

Then $A_{n}$ in equation (18) is equal to the sum of residues of

$$
\phi(p)=2 e^{p / 2} I_{n}(p / 2) \bar{f}(p)
$$

in $L_{c}$.

Proof. Consider the integral

$$
\frac{1}{2 \pi i}\left(\int_{c-i \beta}^{c+i \beta} \phi(p) d p+\int_{\Gamma_{\beta}} \phi(p) d p\right)
$$

where $\phi(p)$ is defined in equation (24), and $\Gamma_{\beta}$ is the semicircle

$$
p=c-\beta e^{i \theta}, \quad-\frac{\pi}{2} \leqq \theta \leqq \frac{\pi}{2} .
$$

We have on $\Gamma_{\beta}$, for large $\beta$ (see reference [6], p. 203),

$$
\left|e^{p / 2} I_{n}(p / 2)\right| \sim|\pi p|^{-1 / 2}\left|e^{p}+i(-1)^{n}\right| \leqq|\pi p|^{-1 / 2}\left(e^{c-\beta \cos \theta}+1\right) .
$$

Hence, by equation (23),

$$
\left|\int_{\Gamma_{\beta}} \phi(p) d p\right| \leqq \pi^{-1 / 2} \operatorname{Max}_{\Gamma_{\beta}}\left|p^{1 / 2} \bar{f}(p)\right|\left(e^{c}+1\right) \rightarrow 0 \quad \text { as } \quad \beta \rightarrow \infty,
$$

and the result follows.

As an example, consider $f(x)=e^{k^{2} x}$ for which $\bar{f}(p)=\left(p-k^{2}\right)^{-1}$. Theorem 1 gives immediately that

$$
A_{n}=2 e^{k^{2} / 2} I_{n}\left(k^{2} / 2\right)
$$

If we write $x=\xi^{2}$, then, since $T_{n}{ }^{*}\left(\xi^{2}\right)=T_{2 n}(\xi)$, we obtain

$$
e^{k^{2} \xi^{2}}=2 \sum_{n=0}^{\infty} e^{k^{2} / 2} I_{n}\left(k^{2} / 2\right) T_{2 n}(\xi),
$$

valid for $-1 \leqq \xi \leqq 1$.

In the next section we shall consider the more interesting problem of expansions in series of ordinary Chebyshev polynomials $T_{n}(x)$.

5. Laplace Transform; Expansion in $T_{n}(x)$. To obtain a formula analogous to equation (22) for the coefficients $a_{n}$ defined in equation (1), we note that the inversion integral in equation (20) gives 0 for $x<0$ and $\frac{1}{2} f(0)$ for $x=0$. To account for negative values of $x$, we define two functions $f_{1}(x)$ and $f_{2}(x)$ as follows:

$$
f_{1}(x)=\left\{\begin{array}{ll}
f(x), & x>0, \\
\frac{1}{2} f(0), & x=0, \\
0, & x<0,
\end{array} \quad f_{2}(x)= \begin{cases}f(x), & x<0, \\
\frac{1}{2} f(0), & x=0, \\
0, & x>0 .\end{cases}\right.
$$

Then, for all real $x$, we have

$$
f(x)=f_{1}(x)+f_{2}(x) .
$$


Define

$$
\begin{aligned}
& \bar{f}_{1}(p)=\int_{0}^{\infty} e^{-p x} f(x) d x=\mathfrak{L}\{f(x)\} \\
& \bar{f}_{2}(p)=\int_{0}^{\infty} e^{-p x} f(-x) d x=\mathscr{L}\{f(-x)\} .
\end{aligned}
$$

Then by the inversion theorem (equation (20)), we have

$$
f_{1}(x)=\frac{1}{2 \pi i} \lim _{\beta \rightarrow \infty} \int_{c_{1}-i \beta}^{c_{1}+i \beta} e^{p x} \bar{f}_{1}(p) d p,
$$

and

$$
f_{2}(-x)=\frac{1}{2 \pi i} \lim _{\beta \rightarrow \infty} \int_{c_{2}-i \beta}^{c_{2}+i \beta} e^{p x} \bar{f}_{2}(p) d p,
$$

where $c_{1}, c_{2}$ are chosen so that all singularities of $\bar{f}_{i}(p), i=1,2$, are to the left of the vertical line through $c_{i}$.

Writing $x$ for $-x$ and replacing $p$ by $-p$ in equation (30) we have, for $x<0$,

$$
f_{2}(x)=\frac{1}{2 \pi i} \lim _{\beta \rightarrow \infty} \int_{-c_{2}-i \beta}^{-c_{2}+i \beta} e^{p x} \bar{f}_{2}(-p) d p .
$$

Thus all singularities of $\bar{f}_{2}(-p)$ are to the right of the line through $-c_{2}$. Combining these results we have, for all $x$,

$$
f(x)=\frac{1}{2 \pi i} \lim _{\beta \rightarrow \infty}\left\{\int_{c_{1}-i \beta}^{c_{1}+i \beta} e^{p x} \bar{f}_{1}(p) d p+\int_{-c_{2}-i \beta}^{-c_{2}+i \beta} e^{p x} \bar{f}_{2}(-p) d p\right\} .
$$

In particular, if, for the range $-1 \leqq x \leqq 1, f(x)$ is given by equation (1), then, since ([6], p. 369)

$$
e^{p x}=2 \sum_{n=0}^{\infty} I_{n}(p) T_{n}(x)
$$

we have on substituting equations (1) and (33) into equation (32), the required result,

$$
a_{n}=\frac{1}{\pi i} \lim _{\beta \rightarrow \infty}\left\{\int_{c_{1}-i \beta}^{c_{1}+i \beta} I_{n}(p) \bar{f}_{1}(p) d p+\int_{-c_{2}-i \beta}^{-c_{2}+i \beta} I_{n}(p) \bar{f}_{2}(-p) d p\right\},
$$

where $\bar{f}_{1}(p)=\mathscr{L}\{f(x)\}$ and $\bar{f}_{2}(p)=\mathscr{L}\{f(-x)\}$.

In the next section we shall illustrate the evaluation of these line integrals by means of a few examples.

6. Examples. A simple illustration is given by considering the function $f(x)=e^{a x}$ where $a>0$. Then $\bar{f}_{1}(p)=1 /(p-a)$ and $\bar{f}_{2}(p)=-1 /(p-a)$. The constants $c_{1}, c_{2}$ have to be chosen so that $c_{1}>a$ and $c_{2}<a$. Equation (34) gives

$$
a_{n}=\frac{1}{\pi i} \lim _{\beta \rightarrow \infty}\left\{\int_{c_{1}-i \beta}^{c_{1}+i \beta}+\int_{c_{2}+i \beta}^{c_{2}-i \beta}\right\} \frac{I_{n}(p)}{p-a} d p .
$$


The integrand tends to 0 when $\beta \rightarrow \pm \infty$ in $p=c+i \beta, c_{2}<c<c_{1}$. Hence, by the theorem of residues, $a_{n}=2 I_{n}(a)$, as required.

A rather more interesting example is given by the function $f(x)=(1 / x) J_{1}(k x)$ which has been tabulated by Clenshaw [5] for $k=8$. We have $\bar{f}_{1}(p)=(1 / k)$. $\left(\sqrt{ }\left(p^{2}+k^{2}\right)-p\right)$ and $\bar{f}_{2}(-p)=-\bar{f}_{1}(p)$. As before, the integrand tends to 0 when $\beta \rightarrow \pm \infty$ in $p=c+i \beta$ and, hence,

$$
a_{n}=\frac{1}{k \pi i} \int_{C} I_{n}(p)\left(\sqrt{ }\left(p^{2}+k^{2}\right)-p\right) d p,
$$

where $C$ is any contour enclosing the slit from $-i k$ to $+i k$. By displacing the contour to the slit we find that

$$
a_{n}=\frac{2 i^{n}}{k \pi} \int_{-k}^{k} J_{n}(y) \sqrt{ }\left(k^{2}-y^{2}\right) d y .
$$

This integral is zero when $n$ is odd and, when $n$ is even $(=2 m)$, we have

$$
a_{2 m}=\frac{4(-1)^{m}}{k \pi} \int_{0}^{k} J_{2 m}(y) \sqrt{ }\left(k^{2}-y^{2}\right) d y .
$$

Writing $y=k \cos \theta$ and using the result ([6], p. 150) that

$$
J_{\mu}(z) J_{\nu}(z)=\frac{2}{\pi} \int_{0}^{\pi / 2} J_{\mu+\nu}(2 z \cos \theta) \cos (\mu-\nu) \theta d \theta,
$$

we find that

$$
a_{2 m}=(-1)^{m} k\left\{J_{m}{ }^{2}(k / 2)-J_{m-1}(k / 2) J_{m+1}(k / 2)\right\} .
$$

This result does not seem to have appeared in the literature before.

A similar analysis, using the same contour as before, can be made for the function $f(x)=(1 / x) \sin k x$. Now $\vec{f}_{1}(p)=\arctan (k / p)=(1 / 2 i) \log ((p+i k) /(p-i k))$ and $\bar{f}_{2}(-p)=-\bar{f}_{1}(p)$. We find

$$
a_{2 m}=2(-1)^{m} \int_{0}^{k} J_{2 m}(y) d y=4(-1)^{m} \sum_{r=0}^{\infty} J_{2 m+2 r+1}(k),
$$

and $a_{2 m+1}=0$.

As a further example, we consider the function $e^{-k^{2} x^{2}}$. Then

$$
\begin{aligned}
& \bar{f}_{1}(p)=\frac{\sqrt{ } \pi}{2 a} e^{p^{2} / 4 k^{2}}(1-\operatorname{erf}(p / 2 k)), \\
& \bar{f}_{2}(p)=\frac{\sqrt{ } \pi}{2 a} e^{p^{2} / 4 k^{2}}(1+\operatorname{erf}(p / 2 k)) .
\end{aligned}
$$

Neither $\bar{f}_{1}(p)$ nor $\bar{f}_{2}(-p)$ has singularities and we can choose $c_{1}=c_{2}=0$. Equation (34) gives

$$
a_{n}=\frac{2}{\pi i} \lim _{\beta \rightarrow \infty} \int_{-i \beta}^{i \beta} I_{n}(p) \frac{\sqrt{ } \pi}{2 k} e^{p^{2} / 4 k^{2}} d p
$$


When $n$ is odd, this gives $a_{n}=0$ and, when $n$ is even $(=2 m)$, we find

$$
\begin{aligned}
a_{2 m} & =\frac{2(-1)^{m}}{k \sqrt{ } \pi} \int_{0}^{\infty} J_{2 m}(y) e^{-y^{2} / 4 k^{2}} d y \\
& =2(-1)^{m} e^{-k^{2} / 2} I_{m}\left(k^{2} / 2\right),
\end{aligned}
$$

using the result given in [6], p. 394.

Finally, we can proceed in an exactly analogous way for the function erf $(k x)$. Then,

$$
\bar{f}_{1}(p)=(1 / p) e^{p^{2} / 4 k^{2}}[1-\operatorname{erf}(p / 2 k)] .
$$

Since erf $(k x)$ is an odd function,

and

$$
\bar{f}_{2}(p)=-\bar{f}_{1}(p)
$$

Proceeding as for $e^{-k^{2} x^{2}}$, we find

$$
a_{n}=\frac{2}{\pi i} \lim _{\beta \rightarrow \infty} \int_{-i \beta}^{i \beta} \frac{I_{n}(p)}{p} e^{p^{2} / 4 k^{2}} d p .
$$

Writing $p=i y$, we find

$$
a_{n}=\frac{2(-1)^{n-1}}{\pi} \int_{-\infty}^{\infty} \frac{J_{n}(y)}{y} e^{-y^{2} / 4 k^{2}} d y .
$$

When $n$ is even, $a_{n}$ is zero and when $n$ is odd $(=2 m+1)$, we have

$$
a_{2 m+1}=\frac{2(-1)^{m}}{\pi(2 m+1)} \int_{0}^{\infty}\left[J_{2 m}(y)+J_{2 m+2}(y)\right] e^{-y^{2} / 4 k^{2}} d y,
$$

on using the recurrence relation for the Bessel functions. Evaluating the integral gives

$$
a_{2 m+1}=\frac{2 k(-1)^{m} e^{-k^{2} / 2}}{(\sqrt{ } \pi)(2 m+1)}\left[I_{m}\left(\frac{k^{2}}{2}\right)+I_{m+1}\left(\frac{k^{2}}{2}\right)\right] .
$$

School of Physics

University of Sydney

now at

Mathematics Department

University of Tasmania, Hobart

Tasmania, Australia

School of Mathematics

University of New South Wales

Kensington, N.S.W., Australia.

1. D. Elliotr, "The evaluation and estimation of the coefficients in the Chebyshev series expansion of a function," Math. Comp., v. 18, 1964, p. 274-284.

2. C. W. Clenshaw, "The numerical solution of linear differential equations in Chebyshev series," Proc. Cambridge Philos. Soc., v. 53, 1957, p. 134-149. MR 18, 516.

3. D. ElliotT, "A Chebyshev series method for the numerical solution of Fredholm integral equations," Comput. J., v. 6, 1963 p. 102-111. MR 27 \#5386.

4. N. G. DE BruIJN, Asymptotic Methods in Analysis, North-Holland, Amsterdam, 1958. MR $20 \# 6003$.

5. C. W. Clenshaw, "Chebyshev series for mathematical functions," Nat. Physical Lab. Math. Tables, v. 5, Dept. Scientific \& Indust. Res., H.M.S.O., London, 1962. MR 26 \#362.

6. G. N. Watson, A Treatise on the Theory of Bessel Functions, Cambridge Univ. Press, New York, 1944; 2nd ed., 1952. MR 6, 64. 